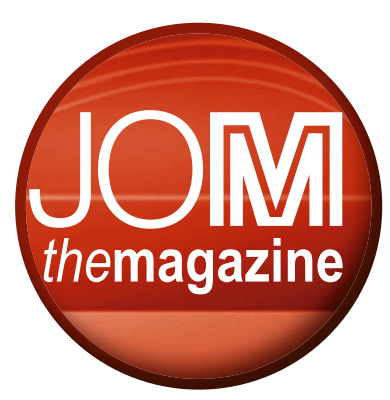

\title{
2020 Peer Reviewers: Thank You for Your Contributions
}

JOM extends a heartfelt thank-you to all volunteers who served as peer reviewers in 2020. This was a difficult year for many, as the Covid-19 virus wreaked havoc on daily life-health, family, work, and more-around the world. The audience and authors of JOM are indebted to these reviewers who, even during a pandemic, helped to ensure that only the highest-quality articles are published.

\begin{tabular}{|c|c|}
\hline Halime Abakay Temel & Desmond Attah-Kyei \\
\hline Abdalla Abdalla & Edgar Avalos Gauna \\
\hline Hani Abdelhamid & Katri Avarmaa \\
\hline Benjamin Adam & Samuel Awe \\
\hline Solomon Afelik & Tolga Aytug \\
\hline Aquil Ahmad & Alafara Baba \\
\hline Mark Aindow & K. Suresh Babu \\
\hline Hamid Akbarzadeh & Sunil Badwe \\
\hline Torgom Akopyan & Ebad Bagherpour \\
\hline Zafir Alam & Emre Bal \\
\hline Miltos Alamaniotis & Shenxu Bao \\
\hline Houshang Alamdari & Nilam Barekar \\
\hline Noe Alba-Baena & Praful Bari \\
\hline Carl Allertz & Dallin Barton \\
\hline Bandar AlMangour & Amrita Basak \\
\hline Richard Alorro & Hymavathi Basireddy \\
\hline Mahmut Altiner & Thomas Battle \\
\hline Srinivasan Anandhan & Behzad Bavarian \\
\hline Alexandra Anderson & Robert Baxter \\
\hline David Andersson & Vishwas Bedekar \\
\hline Stoichko Antonov & Santosh Behera \\
\hline Mahmood Anwar & Bernd-Arno Behrens \\
\hline Fatih Apaydin & Sefiu Bello \\
\hline Diran Apelian & Ivan Belo Fernandes \\
\hline Jokilaakso Ari & Nikolay Belov \\
\hline Raymundo Arroyave & Birgul Benli \\
\hline A. Arul Marcel Moshi & Stéphane Berbenni \\
\hline
\end{tabular}

Somesh Bhattacharya

Dhriti Bhattacharyya

Venkata Bheemreddy

Ce Bian

Zhenzhong Bian

Laurent Birry

Giovanni Bolelli

David Bourell

Richard Bradshaw

Samuel Briggs

Steffen Brinckmann

Stephan Broek

Geoffrey Brooks

Alexander Brosius

Daniel Builes

Mohd Hamdi Bin Ali Buraidah

Osvaldo Camara

Carelyn Campbell

Patrick Cantwell

Qing Cao

Zhanmin Cao

Zhao Cao

John Carpenter

Jose Adilson Castro

Cyril Cayron

Jiajie Cen

Liyuan Chai
Suwaree Chankitmunkong

V S Reddy Channu

Chien-Wei Chao

Indrajit Charit

Alexandros Charitos

Rakesh Chaudhary

Ankur Chauhan

Bingxv Chen

Chih-Ming Chen

Lin Chen

Mao Chen

Mengjun Chen

Mengyuan Chen

Po-Yu Chen

Shaowei Chen

Shiliang Chen

Tianyi Chen

Wei Chen

Xiang Chen

Xiaobo Chen

Yongming Chen

Yunhui Chen

Chu Cheng

Ruihua Cheng

Siyu Cheng

Durgadas Cherukaraveedu

Deside Chibwe 


\begin{tabular}{|c|c|c|c|}
\hline Geetha Chimata & Marc Dupuis & Maryam Ghodrat & Mingsheng He \\
\hline Seongmook Cho & Agnieszka Dybalska & M.K. Ghosh & Edward Herderick \\
\hline Sun Choi & Sven Eckert & Wojciech Gierlotka & Owen Hildreth \\
\hline Diana Cholico & Benjamin Eftink & Gary Gladysz & Cheng-En Ho \\
\hline Dianne Chong & Ahmed El-Gendy & Manmohan Goel & Lauri Holappa \\
\hline Kwok Feng Chong & Richard Elliott & Gultekin Goller & Elizabeth Holm \\
\hline Lokesh Choudhary & Abdallah Elsayed & Jiawei Gong & George Holywell \\
\hline Sugata Chowdhury & Fatih Erdemir & Gonzalo Gonzalez & Peter Hosemann \\
\hline Andrew Chuang & Dinc Erdeniz & Sumedh Gostu & Zahra Hosseini \\
\hline Mateusz Ciszewski & Ihsan Erikat & John Grandfield & L.G. Hou \\
\hline Kester Clarke & Youqi Fan & Michael Grapes & Han-Yu Hsueh \\
\hline James Coakley & Chaojun Fang & Peter Groche & Fang $\mathrm{Hu}$ \\
\hline Eric Cockayne & Gang Fang & Jianzheng Guo & Xiaobing Hu \\
\hline Armando Coronado & Saeed Farrokhpay & Jingdong Guo & Yixin Hua \\
\hline Jun Cui & Qingming Feng & Jinshan Guo & Ke Huang \\
\hline Weisz-Patrault Daniel & Yuqing Feng & John Guo & Peifeng Huang \\
\hline Massimiliano Dapporto & Camille Fleuriault & Lingling Guo & Wei Huang \\
\hline Hrishikesh Das & Kerstin Forsberg & Qilin Guo & Yan Huang \\
\hline Melvin David Kumar & Karl Forwald & Weimin Guo & Yuanding Huang \\
\hline J.D.B. de Mello & Lesley Frame & Xueyi Guo & Yukun Huang \\
\hline Karnika De Silva & Jack Francis & Ashish Kumar Gupta & Liu Hui \\
\hline Johan de Villiers & Michael Francis & Siddharth Gupta & Guangsheng Huo \\
\hline Jiushuai Deng & Min Gan & Turan Gurgenc & Maxwell Hutchinson \\
\hline Zhigan Deng & Kinjal Gandha & Bharat Gwalani & Robert Hyers \\
\hline Sheila Devasahayam & Krishna Ganesan & Amir Hadadzadeh & Happiness lijie \\
\hline Nikhil Dhawan & R. Ganesan & Athir M Haddad & Chukwunwike lloeje \\
\hline Di Di Chen & Bingliang Gao & Ravi Hadimani & Sadia llyas \\
\hline Martin Diehl & Lei Gao & Taher Hajilounezhad & Z lqbal \\
\hline Wang Dihua & Qiuzhi Gao & John Hald & Nurul Ain Jabit \\
\hline Isaac Dinaharan & Yanfei Gao & Sabuj Halder & Alain Jacot \\
\hline Meheretu Jaleta Dirbeba & Akhil Garg & Petteri Halli & Amin Jafari-Ramiani \\
\hline Pei Dong & Maneesha Garg & Morad Hamad & Vijay Kumar Jain \\
\hline Aichun Dou & Andrew Gaynor & Haisheng Han & Saumyadeep Jana \\
\hline Jun Du & Amber Genau & Zhaohui Han & V. Jayasankar \\
\hline Qiang Du & Shujiang Geng & Douglas Hansen & Cheng Ji \\
\hline Wenjia Du & Elizabeth Getto & Sada Haruna & Shouxun Ji \\
\hline Xintong Du & Roger Ghanem & Khalid Hattar & Vincent Ji \\
\hline Huamei Duan & Aimen Gheribi & Jeff Hawk & Jie Jian \\
\hline
\end{tabular}




\begin{tabular}{|c|c|c|c|}
\hline Xin Jiang & S. Shashi Kumar & Feng-Qin Liu & Elias Matinde \\
\hline Kexin Jiao & Sumit Kumar & Guihua Liu & Douglas Matson \\
\hline Shuqiang Jiao & Julia Kundin & Honghui Liu & Hiroyuki Matuura \\
\hline Miaomiao Jin & Richard Kunter & Lei Liu & Michael Maughan \\
\hline Hannu Johto & Hiroyuki Kuwae & Runqing Liu & Tsuyoshi Mayama \\
\hline Andrea Jokisaari & Ohyung Kwon & Tingkun Liu & Scott McCall \\
\hline Pooran Joshi & Sun Kwon & Wei-Ran Liu & Scott McCormack \\
\hline Sameehan Joshi & Vittoria Laghi & Wenying Liu & David McDowell \\
\hline Shrikant Joshi & Mehdi Lalpoor & Xuheng Liu & James McGuffin-Cawley \\
\hline Vineet Joshi & Chiranjivi Lamsal & Yu-chen Liu & Alexander McLean \\
\hline John Joyce-Rotella & Lihui Lang & Zhiqiang Liu & Pedro Medeiros Ribeiro \\
\hline William Judge & André Fabián Lasagni & Javier LLorca & Alexander Medvedev \\
\hline Jiheon Jun & Petri Latostenmaa & Mujun Long & Andreas Mehner \\
\hline Jae-Young Jung & Julien Lauzon-Gauthier & Raymond Longbottom & Abhishek Mehta \\
\hline Joshua Kacher & Jaime Lazaro Nebreda & Xiaoyuan Lou & Q. S. Mei \\
\hline Surya Kalidindi & Gerard Lebon & Shenglu Lu & Chamini Mendis \\
\hline Rajamohan Kalluru & Jonghyun Lee & Yan Lu & Yifei Meng \\
\hline Jidong Kang & Kee Sung Lee & Edward Lui & Yujie Meng \\
\hline Chandrakala Kari & Myoung-Gyu Lee & Alan Luo & Benoit Merle \\
\hline Shravan Katakam & Sheng-Long Lee & Dung Luong & Andreas Meyer \\
\hline Changhong Ke & Eberhard Lehmann & Qing Lv & Roland Mežibrický \\
\hline Leslie Kerby & Bing Li & Baozhong Ma & Victoria Miller \\
\hline Michael Kesler & Guanghui Li & Jing Ma & Saereh Mirzababaei \\
\hline Mohammad Umar & Hongyi Li & Xiaodong Ma & Hamed Mirzadeh \\
\hline Farooq Khan & Qiang Li & Yongbo Ma & Brajendra Mishra \\
\hline Parnian Kiani & Weiguan Li & Donald MacKenzie & Kishlay Mishra \\
\hline Volkan Kilicli & Xiao Li & Jonathan Madison & Nur Farhana Diyana Mohd Yunos \\
\hline Hojong Kim & Yang Li & Mohammad Maghsoudi Ganjeh & Mohammad Ali Mohtadi-Bonab \\
\hline K. B. Kim & Ying Li & Shooka Mahboubi & David Molenaar \\
\hline Young Min Kim & Yujie Li & Reza Mahmoodian & Ashok Mondal \\
\hline Mehmet Koç & Guang-Xing Liang & Petra Maier & Kunal Mondal \\
\hline Nagaraja Kodihalli & Zhi Liang & Ali Maleki & Kazuki Morita \\
\hline Sergey Komarov & Mathias Liewald & Keyou Mao & Mykola Moroz \\
\hline Yong Lin Kong & Daniel Lindberg & Dimitrios Maroudas & Yaroslav Mudryk \\
\hline Hiren Kotadia & Bingbing Liu & Aiden Martin & Kalenda Mutombo \\
\hline Tomohisa Kumagai & Chao Liu & Caleb Massey & Naresh Nadammal \\
\hline Deepoo Kumar & Donghui Liu & Ioannis Mastorakos & Soumya Nag \\
\hline Kuldeep Kumar & Fengchao Liu & Glenn Mather & Srinidhi Nagaraja \\
\hline
\end{tabular}




\begin{tabular}{|c|c|c|c|}
\hline Taiki Nakata & Giacomo Po & Maryam Salari & Aaron Stebner \\
\hline Peeyush Nandwana & Reeju Pokharel & Mobin Salasi & Matthew Steiner \\
\hline Mahmoud Nasrollahzadeh & E. Polatidis & Ali Salifu & David St. John \\
\hline Neale Neelameggham & Antti Porvali & Georges Salloum-Abou-Jaoude & Krzysztof Stopka \\
\hline Van Nguyen & Adam Powell & Imam Santoso & Zijian Su \\
\hline Stuart Nicol & Arvind Prasad & Taisuke Sasaki & Tungky Subroto \\
\hline Changning Niu & Leon Prentice & Mark Schlesinger & Chantal Sudbrack \\
\hline Jianbing Niu & Paul Prichard & Michael Schmidt & Dmitry Sukhomlinov \\
\hline Tongjun Niu & Saurabh Puri & Stephan Schneider & Cheng Sun \\
\hline Yanxia Niu & Jonathan Puthoff & Carsten Schwandt & Veeraraghavan Sundar \\
\hline Mamdouh Omran & Ma Qian & Samuel Senanu & Kumar Sundaram \\
\hline Richard Otis & Jiadong Qin & Zhongxia Shang & Ryosuke Suzuki \\
\hline Harald Øye & Jiayong Qiu & Prabhanshu Shekhar & D. Swinbourne \\
\hline Cihan Ozel & Haozheng Qu & Leiting Shen & Martin Syvertsen \\
\hline Tayfur Ozturk & Krishna Rajan & Baodong Shi & Aaron Tallman \\
\hline Ali Tuncay Ozyilmaz & Pavel Ramirez Lopez & Ge Shi & Keqin Tan \\
\hline Min-Kyu Paek & Vijaya Rangari & Wenwu Shi & Chaobo Tang \\
\hline Xiaolin Pan & Mingjun Rao & Kwang Seon Shin & Xianzhong Tang \\
\hline Stefanos Papanikolaou & Subba Rao & Denis Shishin & Zhenghua Tang \\
\hline James Paramore & Benjamin Ravary & Malgorzata Sikora-Jasinska & Pekka Taskinen \\
\hline Changyong Park & Nuggehalli Ravindra & Wim Sillekens & Fiseha Tesfaye \\
\hline Jun-Sang Park & Sudhir Ravula & Alok Singh & Jayson Tessier \\
\hline Deepak Patel & Guillaume Reinhart & Ashish Singh & Dan Thoma \\
\hline Tanaji Paul & Chai Ren & Jitendra Kumar Singh & Lei Tian \\
\hline Noah Paulson & Natalia Resnina & Shiril Sivan & lain Todd \\
\hline Gorakh Pawar & Marcus Rettenmayr & Ana Socalici & Mingming Tong \\
\hline Dong Peng & Md. Reza-E-Rabby & Faraz Soltani & Catherine Tonry \\
\hline Hong Peng & M. Akbar Rhamdhani & Antonio Sombra & Mahdi Torabi Rad \\
\hline Yawei Peng & Manuel Roldan & Bo Song & Ha Trinh \\
\hline Florian Markus Penz & Anthony Rollett & Jianxun Song & Prabhat Tripathy \\
\hline Linus Perander & Hans Jørgen Roven & Tingting Song & Rahul Trivedi \\
\hline Ray Peterson & David Rowenhorst & Leila Sorkhi & Mark Tschopp \\
\hline Frank Pfefferkorn & Kyle Rozman & Abiraman Srinivasan & Lefteri Tsoukalas \\
\hline Simon Phillpot & Timothy Rupert & Raghu Srinivasan & David Tweddle \\
\hline Dinesh Pinisetty & Jon Ryu & Sharada Srinivasan & Huseyin Ucar \\
\hline Bruce Pint & James Saal & Prakash Srirangam & Begum Unveroglu \\
\hline Petrus Pistorius & Rawia F. Sadek & Rajiv Srivastava & Maria Beatriz Valcarce \\
\hline Alex Plotkowski & Guðrún Sævarsdóttir & Marius Stan & Maurits Van Camp \\
\hline
\end{tabular}




\begin{tabular}{|c|c|c|c|}
\hline James Vaughan & Yandong Wang & Yong Yang & Long Zhang \\
\hline Dirk Verhulst & Yunbo Wang & John Yeager & Mingxing Zhang \\
\hline Jose Victoria-Hernandez & Zhenyang Wang & Yee-wen Yen & Ning Zhang \\
\hline N. Vijay Ponraj & Charles Ward & Jingjie Yeo & Teng Zhang \\
\hline Vineeth Vijayan & Martin Weber & Yaolin Yi & Ting-an Zhang \\
\hline Vlastimil Vodarek & Tzu-Chien Wei & Huayi Yin & Weiguang Zhang \\
\hline Travis Voorhees & Timothy Weihs & Kazuharu Yoshizuka & Yifan Zhang \\
\hline Hitesh Vora & Christopher Weinberger & Hang Yu & Yijie Zhang \\
\hline Bey Vrancken & Janelle Wharry & Yaowei Yu & Ying Zhang \\
\hline Katia Vutova & Robert Wheeler & Lang Yuan & Yingyan Zhang \\
\hline Adrian Wagner & Thomas Wolfe & Tian-Feng Yuan & Yingyi Zhang \\
\hline Chao Wang & David Wong & YiYuan & Yuanbo Zhang \\
\hline Chengpeng Wang & Chenglin Wu & Mohammad Zamanzade & Yunhu Zhang \\
\hline Chengyan Wang & Hsin-jay Wu & Ximin Zang & Zhiliang Zhang \\
\hline Cong Wang & Menghuai Wu & Giovanni Zangari & Ding Zhao \\
\hline Fei Wang & Longgong Xia & Alexander Zavrazhnov & Hongliang Zhao \\
\hline Gang Wang & Liming Xiong & Steven Zeltmann & Jiuzhou Zhao \\
\hline Jianfeng Wang & Xianbo Xu & Baogang Zhang & Yangyang Zhao \\
\hline Jingxiu Wang & Zhenming Xu & Baoguo Zhang & Junchao Zheng \\
\hline Lifei Wang & Zhiping Xu & Dalong Zhang & Huamin Zhou \\
\hline Michael Cai Wang & Wentao Yan & Duyao Zhang & Jie Zhou \\
\hline Mingyu Wang & Chao Yang & Guangzong Zhang & Lejun Zhou \\
\hline Qi Wang & Congren Yang & Haisong Zhang & Qiang Zhu \\
\hline Shujuan Wang & Ge Yang & Hui Zhang & Steven Zinkle \\
\hline Xiangwen Wang & Hongying Yang & Jialiang Zhang & Olga Zinovieva \\
\hline Xianqiao Wang & Jianguang Yang & Lei Zhang & James Zuback \\
\hline Xiaodong (Alice) Wang & Shufeng Yang & Liang Zhang & \\
\hline Yan Wang & Yafeng Yang & Lifeng Zhang & \\
\hline
\end{tabular}

